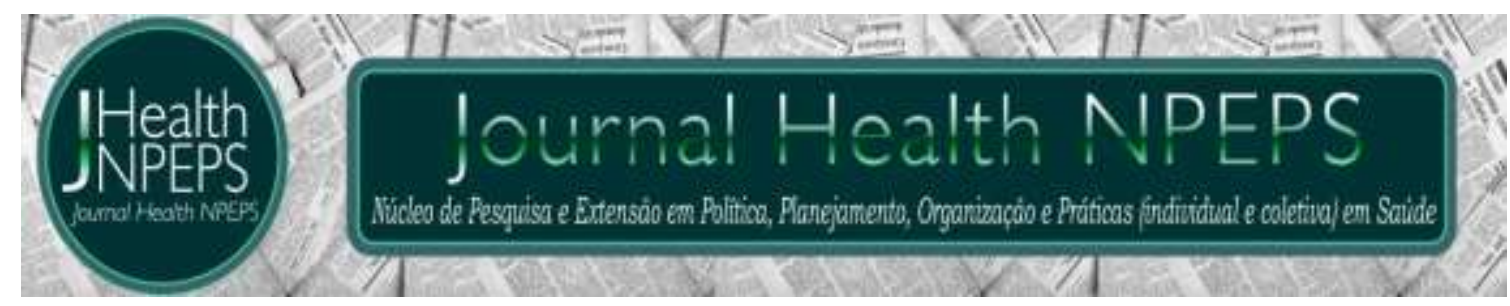

http://dx.doi.org/10.30681/252610103378

ARTIGO DE REVISÂO

\title{
Produção de conhecimento em enfermagem de reabilitação portuguesa - scoping review
}

\section{Knowledge production in portuguese rehabilitation nursing - scoping review}

\section{Producción de conocimiento en enfermería de rehabilitación portugués - scoping review}

\section{Carla Sílvia Fernandes ${ }^{1}$, José Augusto Gomes ${ }^{2}$, Bruno Miguel Magalhães ${ }^{3}$, Andreia Maria Novo Lima $^{4}$}

\section{RESUMO}

Objetivo: mapear a produção de conhecimento em enfermagem de reabilitação em periódicos nacionais e internacionais. Método: revisão do tipo scoping, com base nos princípios preconizados pelo "Joanna Briggs Institute". Os estudos foram extraídos com base nos critérios de inclusão e exclusão. Foram considerados como critérios de inclusão as publicações em periódicos nacionais e internacionais de autoria ou coautoria de enfermeiros portugueses, sem limites temporais. Foram excluídos os documentos cujo foco de intervenção não fosse a enfermagem de reabilitação. Resultados: foram considerados 29 estudos publicados entre 1979 e 2018, sendo possível identificar três áreas temáticas relevantes: Transições Saúde-doença, Promoção Saúde e Qualidade em Enfermagem de Reabilitação. Os resultados fundamentam a área de intervenção do enfermeiro de reabilitação mais evidente no âmbito das transições saúde-doença. Conclusão: apesar do aumento da publicação científica nos últimos três anos sobre essa temática,

\footnotetext{
${ }^{1}$ Enfermeira. Pós-doutora em Enfermagem. Professor Auxiliar na Escola Superior de Saúde- Universidade Fernando Pessoa. Porto, Portugal, Portugal. E-mail: carlasilviaf@gmail.com ORCID ID: http://orcid.org/0000-0001-7251-5829 Autor principal - Endereço para correspondência: Praça 9 de Abril 349, 4249-004, Porto, Portugal.

${ }^{2}$ Enfermeiro. Mestre em Enfermagem de Reabilitação. Enfermeiro na Clínica Nossa Senhora da Guia- Vila do Conde, Portugal. E-mail: japgomes@gmail.com ORCID ID: http://orcid.org/0000-0002-7486-1378

${ }^{3}$ Enfermeiro. Mestre em Saúde Pública. Professor Adjunto na Escola Superior de Saúde Santa Maria. Porto, Portugal. E-mail: bruno.magalhaes@santamariasaude.pt ORCID ID: http://orcid.org/0000-0001$\underline{6049-8646}$

${ }^{4}$ Enfermeira. Mestre em Enfermagem de Reabilitação. . Professor Auxiliar na Escola Superior de SaúdeUniversidade Fernando Pessoa. Porto, Portugal. E-mail: amlima@ufp.edu.pt ORCID ID: http://orcid.org/0000-0001-7535-9040
} meio, desde que a publicação original seja corretamente citada. 
envolvendo a enfermagem de reabilitação, é necessário continuar desenvolvendo estudo nesse campo, a fim de fortalecer as evidências sobre esse cuidado de saúde.

Descritores: Reabilitação; Enfermagem em Reabilitação; Enfermagem.

\section{ABSTRACT}

Objective: to map knowledge production in rehabilitation nursing in national and international journals. Method: scoping-type review, based on the principles advocated by the "Joanna Briggs Institute". The studies were extracted based on the inclusion and exclusion criteria. Inclusion criteria were publications in national and international journals authored or coauthored by Portuguese nurses, with no time limits. The documents in which focus was not rehabilitation nursing were excluded. Results: 29 studies published between 1979 and 2018 were considered, and it was possible to identify three relevant thematic areas: Health-disease transitions, Health Promotion and Quality in Rehabilitation Nursing. The results support the area of intervention of the rehabilitation nurse, being more evident in the scope of health-disease transitions. Conclusions: despite the increase in scientific publication in the last three years on this theme, involving rehabilitation nursing, it is necessary to continue developing studies in this field in order to strengthen the evidence on this health care.

Descriptors: Rehabilitation; Nursing in Rehabilitation; Nursing.

\section{RESUMEN}

Objetivo: mapear la producción de conocimiento en enfermería de rehabilitación en periódicos nacionales e internacionales. Método: revisión del tipo scoping, con base en los principios preconizados por el "Joanna Briggs Institute". Los estudios se extrajeron de acuerdo con los criterios de inclusión y exclusión. Se considerarán criterios de inclusión publicaciones en revistas nacionales e internacionales de autoría o co-autoría de enfermeros portugueses sin límites de tiempo. Se excluyeron los documentos cuyo foco de intervención no fuera la enfermería de rehabilitación. Resultados: fueron considerados 29 estudios publicados entre 1979 y 2018, siendo posible identificar tres áreas temáticas relevantes: Transiciones Salud-enfermedad, Promoción Salud y Calidad en Enfermería de Rehabilitación. Los resultados fundamentan el área de intervención del enfermero de rehabilitación más evidente en el ámbito de las transiciones salud-enfermedad. Conclusión: a pesar del aumento de la publicación científica en los últimos tres años sobre esa temática, involucrando a la enfermería de rehabilitación es necesario continuar desarrollando estudio en ese campo, a fin de fortalecer las evidencias sobre ese cuidado de salud.

Descriptores: Rehabilitación, Enfermería en Rehabilitación, Enfermería.

INTRODUÇÃO

$\begin{array}{ccccc}\text { A enfermagem é uma } & \text { desenvolvimento da investigação }{ }^{1} \text {. } \\ \text { ciência em construção que vem } & \text { Tem-se sugerido que o }\end{array}$

procurando a consolidação de um espaço de saber a partir do Tem-se sugerido que 0 
conhecimento científico é comunicado aos membros de uma profissão através da sua literatura e, como tal, os periódicos representam um método importante para a divulgação de resultados de pesquisa para a enfermagem ${ }^{2}$. Na última década houve aumento exponencial do número de artigos de enfermagem publicados, com influência na prática profissional de enfermagem ${ }^{3}$.

São inestimáveis as contribuições da investigação científica para o desenvolvimento do campo da enfermagem ${ }^{4}$. 0 Conselho Internacional de Enfermagem refere que a pesquisa em enfermagem, e respectiva divulgação é necessária para gerar novos conhecimentos, avaliar práticas e serviços existentes, e fornecer evidências para o ensino, prática, pesquisa e gestão de enfermagem, salientando que só assim será possível garantir o avanço da profissão ${ }^{5}$.

Estes aspetos são determinantes e imprescindíveis designadamente na estruturação do corpo de conhecimentos Especializados em Enfermagem como é o caso da Reabilitação, aspecto esse evidenciado pela Ordem dos enfermeiros e colégio da respectiva especialidade ${ }^{6,7}$.

O exercício profissional da enfermagem de reabilitação, como área especializada da Enfermagem, tem como alvo a pessoa com necessidades especiais ao longo do ciclo vital, também ela detém uma área de intervenção especializada que decorre de um corpo de conhecimentos e procedimentos específicos $^{6}$. A enfermagem de reabilitação é uma especialidade em crescente prosperidade em alguns países, o que não é o caso em todas as partes do mundo ${ }^{8}$. A Reabilitação é uma prática de especialidade que foi organizada como uma especialidade de enfermagem em 1964 nos EUA ${ }^{9}$.

A enfermagem de reabilitação surgiu formalmente associado às companhias de seguros que contratavam enfermeiros para realizar avaliações de sinistrados. 0 surgimento de enfermagem de reabilitação pode também ser creditado a visionárias como Alice Morrissey, autora do primeiro livro didático no campo, intitulado “Enfermagem de Reabilitação” e 
Harriet Lane, a primeira enfermeira contratado pela Liberty Mutual Insurance Company ${ }^{\circledR} \quad$ para coordenar programas de reabilitação; e Mary A. Mikulic, uma das primeiras especialistas ${ }^{10}$.

Em Portugal, a enfermagem de reabilitação nasceu na década de 1960, com a enfermeira Sales Luís a assumir a responsabilidade da formação dos novos enfermeiros de reabilitação; surgiu o primeiro curso de enfermagem de reabilitação em 18 de outubro de $1965^{6}$. Desde essa altura, a enfermagem de reabilitação tem crescido e consolidado o seu campo de ação nos cuidados de saúde ${ }^{6,9}$.

Para o desenvolvimento desse campo de ação é preponderante que todos os enfermeiros de reabilitação ajudem a disseminar o conhecimento de enfermagem de reabilitação. Isso pode ser realizado de diversas formas, na prática clínica, ensino e gestão. As estratégias de disseminação incluem publicações acadêmicas de resultados de investigação, apresentações em conferências, participação em comitês nacionais e internacionais, ensino de estudantes ou partilha de conhecimento dentro de uma organização profissional ${ }^{11}$.

Sendo a investigação um processo sistemático de criação de conhecimento, cada enfermeiro especialista de enfermagem de Reabilitação deve potenciar o desenvolvimento da própria profissão através da publicação dos resultados da investigação que realiza. Ciente da importância dos aspetos referenciados pretende-se ao longo deste artigo dar resposta à seguinte questão de revisão: Qual o conhecimento científico produzido pela enfermagem de reabilitação em periódicos nacionais $e$ internacionais?, com o objetivo de mapear a produção de conhecimento em enfermagem de reabilitação em periódicos nacionais e internacionais.

\section{MÉTODO}

0 presente estudo de revisão do tipo scoping foi realizado em outubro de 2018. Existem algumas diferenças entre este tipo de revisão e uma revisão sistemática, sendo uma abordagem relativamente nova para a síntese de evidências ${ }^{12}$. As revisões do tipo 
scoping são ferramentas ideais para determinar o corpo de conhecimento sobre um determinado tópico e dar indicação clara do volume de literatura e estudos disponíveis, bem como visão geral de seu conteúdo ${ }^{13}$. Além disso, uma revisão do tipo scoping é mais ampla e flexível do que uma revisão sistemática.

Os pesquisadores descrevem as etapas da revisão de escopo como identificação e apresentação da questão principal de pesquisa, estratégia de busca, identificação dos estudos, seleção dos estudos apropriados, extração dos dados, apresentação, discussão dos dados e divulgação dos resultados $^{12,14}$.

A opção específica pela realização de uma scoping review fundamenta-se por este ser um tipo de revisão que permite mapear evidências em relação ao tempo de publicação, localização, origem, identificando lacunas nas evidências disponíveis sendo essa a finalidade deste percurso ${ }^{14}$.

No sentido de facilitar a definição da pergunta de partida foi utilizada a mnemónica PEO (População, Exposição de interesse e Outcome). Os critérios de inclusão tiveram como principal finalidade orientar a pesquisa e a seleção da literatura científica atual, de modo a aumentar a precisão dos resultados face à questão identificada.

Com base na questão norteadora, a busca dos artigos foi realizada, nas seguintes bases de dados: Medical Literature Analysis and Retrieval System Online (MEDLINE $®$ ), Cumulative Index to Nursing and Allied Health Literature (CINAHL $\left.{ }^{\circledR}\right), \quad$ Psychology and Behavioral Sciences Collection ${ }^{\circledR}$ e na Scientific Electronic Library Online (SciELO). As pesquisas foram realizadas com recurso aos descritores e termos que se seguem para a construção das frases booleanas ((("nurse") OR ("nurs") $O R$ ("rehabilitation nursing") $O R$ ("nursing rehabilitation") $O R$ ("Association of Rehabilitation Nurses") OR ("nursing interventions") $O R$ ("Nursing Role") $O R$ ("nurse practitioner") $O R$ ("nursing care")) AND (("rehabilitation") $\quad O R$ ("restoration") $O R$ ("rehab") $O R$ ("recovery program") $O R$ ("functional program") $O R$ 
("functional exercise") OR

("functional restoration") $O R$

Dos $955 \quad$ estudos

("functional rehabilitation") $O R$

("recovery of function") OR

(“cardiorespiratory rehabilitation")

OR ("rehabilitation program")) AND ((“Portug*”) OR (“Portugal"))).

A identificação dos artigos

a incluir nesta revisão da literatura passou por um processo de seleção rigoroso e sistematizado apresentado no diagrama de Prisma ${ }^{\circledR}$ (Preferred Reporting Items for Systematic Review and MetaAnalyses) que exibe o processo de busca, de exclusão e de seleção dos estudos encontrados. Todo este processo foi realizado pelos investigadores, de forma independente, e o resultado final foi obtido após reunião de consenso. Com vista a sistematizar os dados extraídos dos artigos, foram compilados de forma encontrados através da pesquisa científica realizada, após as diferentes etapas espelhadas na figura 1 foram incluídos 32 estudos. $\mathrm{Na}$ Quadro 1 é realizada a sumarização dos artigos que compuseram a revisão com informação sobre autores, ano de publicação, objetivo, delineamento, amostra, tipo de estudo e amostra.

Em relação aos anos de publicação dos artigos, compreendidos entre 1979 e 2016, os dados coletados apresentaram a distribuição apresentada na Figura 2, evidenciando aumento de produção científica nos últimos anos.

Apesar da referência ao estudo de 1979 da Enfermeira Luís Sales "O enfermeiro de reabilitação descritiva em quadros e figuras.

Quadro 1 - Critérios de inclusão dos artigos científicos a selecionar.

\begin{tabular}{|c|l|}
\hline Critérios de seleção & \multicolumn{1}{|c|}{ Critérios de inclusão } \\
\hline Participantes & Alvo dos cuidados especializados em enfermagem de reabilitação. \\
\hline Exposição & Cuidados de enfermagem de reabilitação. \\
\hline Resultados & $\begin{array}{l}\text { Publicação em periódicos nacionais e internacionais de autoria ou } \\
\text { coautoria de enfermeiros portugueses. }\end{array}$ \\
\hline Data da publicação & Sem limites temporais. \\
\hline Idioma & Inglês, espanhol e português. \\
\hline Tipos de estudos & Todo o tipo de estudo. \\
\hline
\end{tabular}

RESULTADOS E DISCUSSÃO

e o Hemiplégico"34, observam-se 
duas décadas sem produção científica, existindo um "boom" necessário nos últimos três anos, provavelmente relacionado com a indexação de algumas revistas nacionais, e com as produções acadêmicas decorrentes dos mestrados de Enfermagem de reabilitação com características profissionalizantes.

Conforme nos referem estes autores, num estudo sobre 0 mapeamento da literatura em enfermagem de reabilitação, estes resultados não são surpresa, espelhando a crescente importância dos periódicos e publicações em todas as ciências da saúde?. Em relação aos locais de produção, a maioria dos estudos $(n=16)$ foi publicada em revistas Portuguesas, seguindo-se as revistas Brasileiras $(n=4)$, entre outras.

Em relação à metodologia destaca-se que sete estudos correspondem a revisões de literaturas. Os enfermeiros de reabilitação devem implementar intervenções de enfermagem baseadas na melhor evidência, preenchendo a lacuna entre a evidência e a prática ajudando as pessoas alvos dos cuidados a alcançar seus objetivos ${ }^{11}$. Os enfermeiros da reabilitação precisam estar cientes das evidências atuais e integrar evidências apropriadas dentro de seu nível de proficiência ${ }^{11}$.

Da análise, foi possível identificar três áreas temáticas relevantes: Transições Saúdedoença, Promoção Saúde e Qualidade em enfermagem de Reabilitação.

No âmbito do locus de investigação Transições SaúdeDoença, estes aspetos foram visíveis em 12 dos estudos em análise principalmente no âmbito da pessoa vítima de Acidente Vascular Cerebral $(n=4)$, seguindose outros temas como a lesão medular $(\mathrm{n}=2)$, entre outros. Enquanto enfermeiros de reabilitação estes assumem um papel preponderante na forma podem influenciar os processos de transição ao conceberem uma prática centrada na pessoa e nas suas necessidades reais. Segundo a teoria das transições, um dos objetivos principais da disciplina de enfermagem é assistir a pessoa no seu processo de transição, disponibilizando intervenções 
terapêuticas, designadamente conhecimentos e capacidades que permitam despoletar respostas positivas para a vivência da sua transição ${ }^{44}$.

Estes aspetos vão ao encontro dos pressupostos dos Padrões da Qualidade dos Cuidados Especializados em Enfermagem de Reabilitação, na qual é evidenciado que na orientação da prática, os modelos de autocuidado e das transições revelam-se estruturantes e de excelência para a otimização da qualidade do exercício profissional $^{6}$. E alicerçados também nas recomendações das áreas de investigação prioritárias em enfermagem de reabilitação para 2015-2025, onde sobressaem entre outras: Intervenções autônomas do EEER nas áreas da função motora, função respiratória, função cardíaca, função cognitiva, função sensorial e dor, função eliminação intestinal e vesical e, na deglutição; dependência no autocuidado em contexto domiciliário e capacitação da pessoa e/ou cuidador informal ${ }^{7}$.

Nas temáticas referentes ao locus de investigação promoção da saúde, são evidenciados esses aspetos, na promoção da saúde do idoso $(n=4) \quad$ (riscos, exercício, cinesiterapia respiratória, etc), na educação postural $(n=1)$, na adequação dos fatores ambientais (barreiras arquitetónicas $\mathrm{n}=1$ ), $(n=1)$ e na promoção do exercício físico no doente oncológico $(n=1)$. Dos resultados apresentados, embora, a maioria dos programas de reabilitação se direcionem para a lesão aguda ou doença, é essencial que a enfermeira de reabilitação colabore com a pessoa e a equipe interprofissional para ajudar a promover a saúde e prevenir novas deficiências, bem como gerir a sua doença $e$ incapacidade com a melhor independência no ambiente de vida doméstica e comunidade ${ }^{11}$.

As questões referentes à promoção de ações preventivas e prevenção de complicações são destacadas pelo colégio da especialidade nos seus padrões de qualidade dos cuidados $^{6}$. Este domínio tem como alvo a reabilitação o papel da enfermeira de reabilitação na promoção de um bem através da redução de riscos, prevenção de danos e manutenção da saúde ótima ${ }^{11}$. 
Destaca-se também a importância da intervenção da Enfermagem de Reabilitação nos fatores ambientais, participando ativamente na definição de estratégias que, a nível local ou nacional, promovam a plena integração da pessoa com deficiência6. Este aspeto é enfatizado no estudo destes autores, referindo que 0 enfermeiro de reabilitação deve promover a mobilidade, a acessibilidade e a participação social através de conhecimentos próprios sobre legislação específica, sensibilização da comunidade para práticas inclusivas, identificação e eliminação de barreiras arquitetónicas e emissão de pareceres técnico-científicos sobre estruturas e equipamentos sociais da comunidade 22 .

Por último, no locus de investigação qualidade em enfermagem de reabilitação, emergem estudos no âmbito da validação de instrumentos $(n=2)$, ensino $(n=1)$, conceção de cuidados $(n=1)$, produção de indicadores $(n=3)$ e melhoria contínua da qualidade dos cuidados $(n=1)$. Estes aspetos também são salientados nas recomendações das áreas de investigação prioritárias em enfermagem de reabilitação para 2015-2025, onde ressaltam-se, entre outras: a necessidade de estudos sobre a efetividade das intervenções de EEER, o padrão de qualidade para 0 EEER e a validação de escalas ${ }^{7}$. Os Enfermeiros de reabilitação identificam fatores relacionados à qualidade de cuidados $\mathrm{e}$ contribuem para atividades de qualidade, assumindo um papel de liderança na análise e síntese de dados relacionados a resultados de segurança e qualidade, monitorizando e medindo a eficiência do plano de qualidade em nível organizacional ${ }^{11}$. 0 enfermeiro, com especialidade em reabilitação, precisa fazer emergir os elementos determinantes da qualidade dos seus cuidados, numa perspetiva de melhoria contínua ${ }^{28}$.

Destaca-se que os enfermeiros de reabilitação devem priorizar a utilização de evidências atuais e tecnologia de suporte para fornecer $o$ atendimento ideal ao cliente $^{11}$. A pesquisa em enfermagem é um poderoso meio 
de responder a perguntas sobre intervenções de saúde e encontrar melhores formas de promover a saúde, a prevenção de doenças e serviços de atendimento e reabilitação para pessoas de todas as idades e em diferentes contextos. O conhecimento gerado pela pesquisa em enfermagem deve ser utilizado para desenvolver uma prática baseada em evidências, melhorar a qualidade do atendimento e maximizar a saúde, resultados e custo-efetividade das intervenções de enfermagem ${ }^{5}$. Pelo que, importa referir que todos temos a responsabilidade de compartilhar nosso conhecimento ${ }^{8}$. Como limitação nesta revisão, ressalta-se 0 fato de termos incluído apenas artigos publicados em revistas indexadas nas bases de dados, publicados em inglês, português e espanhol.

\section{CONCLUSÃO}

O objetivo desta scoping review foi mapear a produção de conhecimento em enfermagem de reabilitação em periódicos nacionais e internacionais. Foram assim identificados 29 estudos entre 1979 e 2018. Foi possível reconhecer três áreas temáticas relevantes: Transições Saúdedoença, Promoção Saúde e Qualidade em Enfermagem de Reabilitação.

Apesar do aumento da publicação científica nos últimos três anos, é necessário continuar fomentando estudos nesse campo de ação, a fim de ampliar a consolidação e evidencias desses cuidados de saúde. A este nível é preponderante impulsionar e valorar a formação do enfermeiro, de modo que seja evidenciado tanto em nível nacional como internacional 0 papel $\mathrm{da}$ enfermagem de reabilitação na equipe interdisciplinar.

\section{REFERÊNCIAS}

1. Lino M, Backes VM, Costa M, Lino M. Influências capitalistas na produção do conhecimento em enfermagem. Rev gaúch enferm. 2017; 38(1):e61829.

2. Smith DR. A longitudinal analysis of bibliometric and impact factor trends among the core international journals of 
nursing, 1977-2008. J nurs stud. 2010; 47(12):1491-1499.

3. Dardas LA, Woodward A, Scott J, $\mathrm{Xu} \mathrm{H}$, Sawair FA. Measuring the Social Impact of Nursing Research: An Insight into Altmetrics. J Adv Nurs. 2018.

4. Uysal TA, Hamaratçılar G, Tülü B, Erkin Ö. Nursing students' attitudes toward research and development within nursing: Does writing a bachelor thesis make a difference? Int J Nurs Pract . 2017; 23(2).

5. International Council of Nurses. International Nurses' Day Kit, Closing the gap: from evidence to action. 2012. Available from: http: / /www.old.icn.ch/publica tions/2012-closing-the-gapfrom-evidence-to-action /

6. Ordem dos Enfermeiros (OE). Regulamento dos padrões de qualidade dos cuidados especializados em enfermagem de reabilitação. Lisboa; 2011.

7. Ordem dos Enfermeiros (OE). Áreas investigação prioritárias para a especialidade de enfermagem de reabilitação. Lisboa: Ordem dos Enfermeiros; 2014.
8. MacDonell CM, Mauk KL. Where in the world is rehabilitation nursing? Rehabil Nurs . 2017; 42(4):173-174.

9. Spasser MA, Weismantel A. Mapping the literature of rehabilitation nursing. J Med Libr Assoc. 2006; 94(1):137142.

10. Lehman C. The Specialty Practice of Rehabilitation Nursing: A Core Curriculum. Association of Rehabilitation Nurses, 2017.

11. Vaughn $\mathrm{S}$, Mauk KL, Jacelon $\mathrm{CS}$, Larsen PD, Rye J, Wintersgill W, et al. The Competency Model for Professional Rehabilitation Nursing. Rehabil Nurs. 2016; 41(1):33-44.

12. Abou-Setta AM, Jeyaraman MM, Attia A, Al-Inany HG, Ferri M, et al. Correction: Methods for Developing Evidence Reviews in Short Periods of Time: A Scoping Review. PLOS ONE. 2017; 12(2): e0172372.

13. Munn Z, Peters MDJ, Stern $C$, Tufanaru C, McArthur A, Aromataris E. Systematic review or scoping review? Guidance for authors when choosing between a systematic 
or scoping review approach. BMC Med Res Methodol. 2018; 18(1):143.

14. Peters M, Godfrey C, Mclnerney $P$, Soares CB, Khalil H, Parker D. Methodology for jbi scoping reviews. In Aromataris $\mathrm{E}$, editor, The Joanna Briggs Institute Reviewers manual 2015. South Australia: The Joanna Briggs Institute; 2015.

15. Amaral MT. Encontrar um novo sentido da vida: um estudo explicativo da adaptação após lesão medular. Rev Esc Enferm USP. 2009; 43(3): 573-580.

16. Azevedo P, Gomes B. Efeitos da mobilização precoce na reabilitação funcional em doentes críticos: uma revisão sistemática. Referência. 2015; (5):129-138.

17. Cardoso T, Martins MM, Monteiro MC. Unidade de cuidados na comunidade $\mathrm{e}$ promoção da saúde do idoso: um programa de intervenção. Referência. 2017; (13):103-114.

18. Castro P, Martins MM, Couto G, Reis MG. Terapia por caixa de espelho e autonomia no autocuidado após acidente vascular cerebral: programa de intervenção. Referência. 2018;(17): 95-106.

19. Cruz, A, Tosoli-Gomes, AM, Parreira, PM, de Oliveira, DC. Translation and transcultural adaptation of the hospitalized elderly needs awareness scale (henas) for the portuguese language (brazil and portugal). Aquichan. 2017; 17(4):425-436.

20. Faria A, Martins MM, Dornelles S, Oliveira L. Care path of person with stroke: from onset to rehabilitation. Rev bras enferm. 2017; 70(3):495-503.

21. Ferreira AM, Pierdevara $L$, Ventura IM, Brito AM, Franco JM, Mendonça MG. The Gugging Swallowing Screen: A contribution to the cultural and linguistic validation for the Portuguese context. Referência. 2018; 4(16):85-92.

22. Pereira RS, Martins MM, Gomes B, Schoeller S, Laredo-Aguilera JA, Ribeiro I, et al. Municipalities and the promotion of architectural accessibility. Referência. 2018; 4(18):29-38.

23. Fernandes CS, Martins T, SilvaRocha N, Mendes M. "Jogo da postura": programa de 
intervenção de enfermagem em crianças. Aquichan. 2017; 17(2):195-203.

24. Fonseca C, Correia J, Lopes M, Mendes $F$, Mendes D, Marques $C$, et al. Contributions to the ontology of aging, the sensitive indicators of rehabilitation nursing care, in terms of selfcare, in people with respiratory disorders. Paper presented at the Iberian Conference on Information Systems and Technologies. CISTI; 2018. https: //doi.org/10.23919/CISTI .2018 .8399237

25. Fonseca C, Carretas N, Mendes D, Lopes M, Mendes F, Marques C, et al. A proposal of sensitive indicators of the rehabilitation nursing care of people in the surgical process, to be included in the ontology of aging. Paper presented at the Iberian Conference on Information Systems and Technologies. CISTI; 2018. https: //doi.org/10.23919/CISTI .2018 .8399232

26. Fonseca C, Lista A, Lopes M, Mendes F, David Mendes P, Marques $C$, et al. Dependence in self-care with comorbidity, indicators of nursing care and contributions to an ontology of aging: Systematic review of the literature. Paper presented at the Iberian Conference on Information Systems and Technologies. CISTI; 2018. https://doi.org/10.23919/CISTI .2018 .8399236

27. Fumincelli L, Mazzo A, Martins $\mathrm{JC}$, Henriques $\mathrm{F}$, Orlandin L. Quality of life of patients using intermittent urinary catheterization. Rev latinoam enferm. 2017; 25 (1): 29062910.

28. Gomes B. Contributos da formação para o desenvolvimento de competências na área de enfermagem de reabilitação. Texto \& contexto enferm. 2006; 15(2):193-204.

29. Gomes JA, Martins MM, Gonçalves M, Fernandes CS. Enfermagem de reabilitação: percurso para a avaliação da qualidade em unidades de internamento. Referência. 2012; (8):29-38.

30. Mendes RM, Nunes ML, Pinho JÁ, Rodrigues Gonçalves RB. Organization of rehabilitation 
care in Portuguese intensive care units. Rev bras ter intensiv. 2018; 30(1), 57-63.

31. Gouveia BR, Gouveia ÉR, Ihle A, Jardim HG, Martins MM, Freitas $D L$, et al. The effect of the ProBalance Programme on health-related quality of life of community-dwelling older adults: A randomised controlled trial. Arch gerontol geriatr. 2018; 74:26-31.

32. Gouveia BR, Jardim HG, Martins MM, Gouveia ÉR, Freitas DL, Maia JA, et al. An evaluation of a nurse-led rehabilitation programme (the ProBalance programme) to improve balance and reduce fall risk of community-dwelling older people: A randomised controlled trial. Int $\mathrm{j}$ nurs stud. 2016; 56:1-8.

33. Gouveia B, Jardim H, Martins MM. Cinesioterapia em Reabilitação Gerontológica: uma análise da literatura. Referência. 2013; (11):133142.

34. Luís ML. [The rehabilitation nurse and the hemiplegic patient]. Acta med port. 1979; 1(2):279-289.
35. Malheiro MI, Gaspar MF, Barros L. Training CAMP: Effects of an educational program for selfmanagement, on adolescents with spina bifida. Acad Strat Manag J. 2017; 16(2).

36. Nunes H, Queirós PJ. Doente com acidente vascular cerebral: planeamento de alta, funcionalidade e qualidade de vida. Rev bras enferm. 2017; 70(2):415-423.

37. Rodrigues CP, Dias FM. Quality of life of patients with multiple sclerosis and urinary disorders: validation of Qualiveen. Referência. 2018; 4(18):103111.

38. Rosa NR, Lopes M. Sensorimotor Rehabilitation Nursing in Neonatal Intensive Care Units: Integrative Literature Review. Referência. 2015; 4(7):139-147.

39. Prado ARA, Ramos RL, Ribeiro OM, Figueiredo N, Martins MM, Machado W. Bath for dependent patients: Theorizing aspects of nursing care in rehabilitation.

Rev bras enferm. 2017; 70(6):1337-1342.

40. Preto L, Gomes J, Novo A, Mendes MER, Granero-Molina J. Efeitos de um Programa de 
Enfermagem de Reabilitação na Aptidão Funcional de Idosos Institucionalizados. Referência. 2016; servIV(8):55-63.

41. Garcia $\mathrm{Cl}$, Araújo T. Impact of self-care modeling videos on people with spinal cord injury. Referência. 2016; 4(9):57-64.

42. Preto LS, Morgado F, Rodrigues ME, Pinto A. Quality of life and health of people undergoing median nerve decompression surgery. Referência. 2015; (5):101-108.

43. Fernandes CS, Magalhães BM, Santos C, Martínez Galiano JM.
Walking as an intervention during chemotherapy: integrative review. Referência. 2018; (17):118-130.

44. Meleis A. Theoretical Nursing: Development \& Progress. Philadelphia: Wolters Kluwer Health/ Lippincott Williams \& Wilkins; 2012.

Conflito de interesses: Os autores declaram não haver conflito de interesses.

\section{Participação dos autores:}

- Concepção: Fernandes CA, Gomes JA, Magalhães BM.

- Desenvolvimento: Fernandes CA, Gomes JA, Magalhães BM.

- Redação e revisão: Fernandes CA, Gomes JA, Magalhães $B M$, Lima AMN.

Como citar este artigo: Fernandes CA, Gomes JA, Magalhães BM, Lima AMN. Produção de conhecimento em enfermagem de reabilitação portuguesa - scoping review. J Health NPEPS. 2019; 4(1):282-301. 


\section{APÊNDICE}

Id

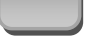

An

EI

In

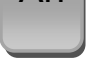

Artigos identificados nas bases de dados eletrónicas: $(n=955)$

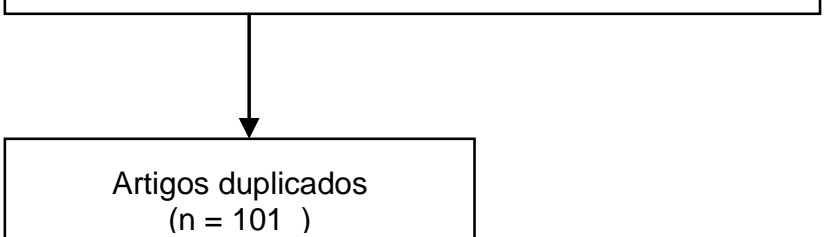

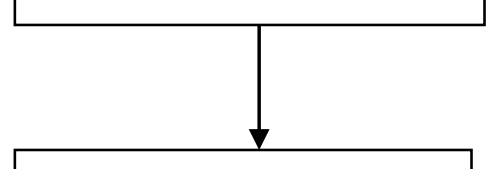

Artigos analisados pelo título e pelo resumo $(n=854)$

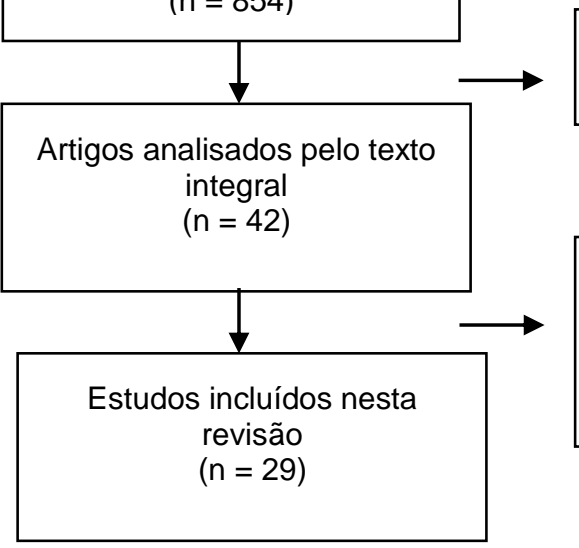

Artigos excluídos $(\mathrm{n}=812)$

Artigos excluídos pelo texto integral 11 = Não são estudos desenvolvidos pela enfermagem de reabilitação $2=$ Repetido

Figura 1 - Fluxograma PRISMA® (adaptado) do processo de seleção dos estudos. Id: identificação, An: análise, EL: elegibilidade, In: inclusão.

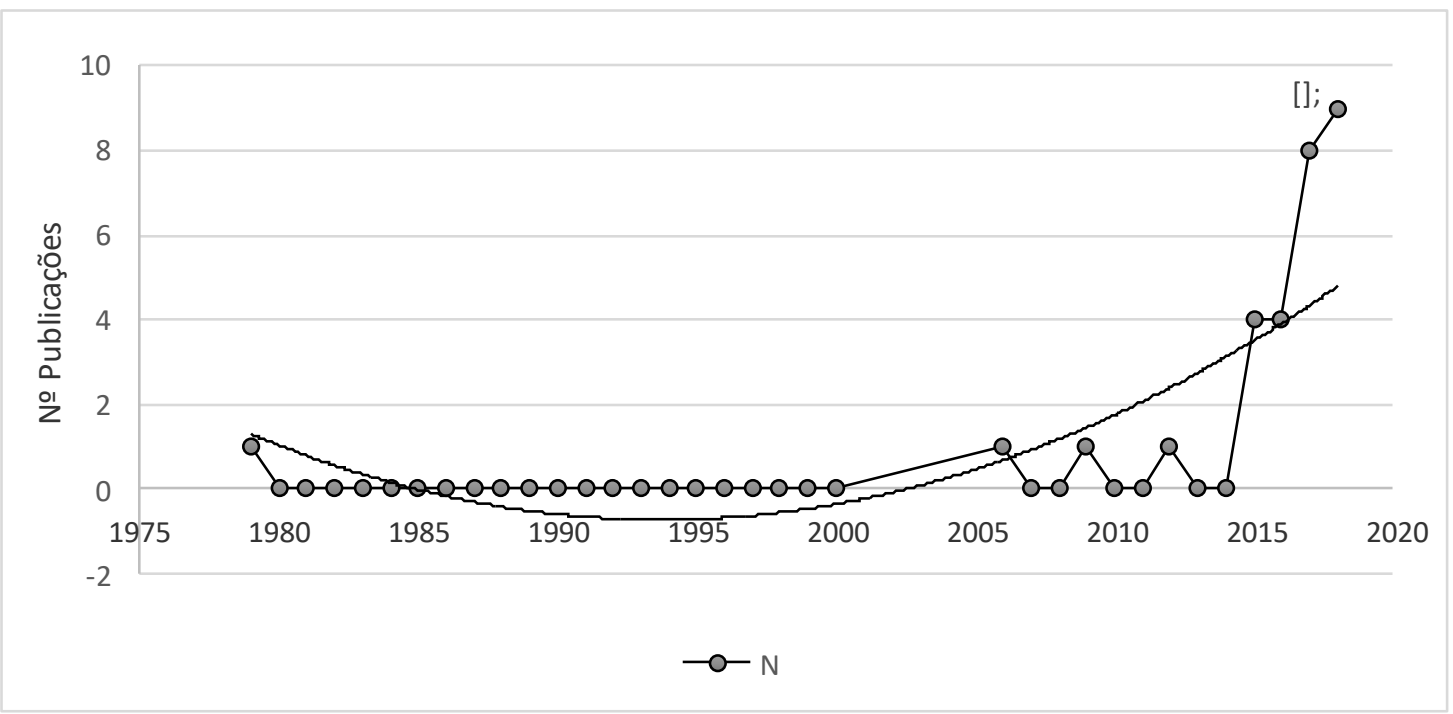

Figura 2 - Publicações por ano. 


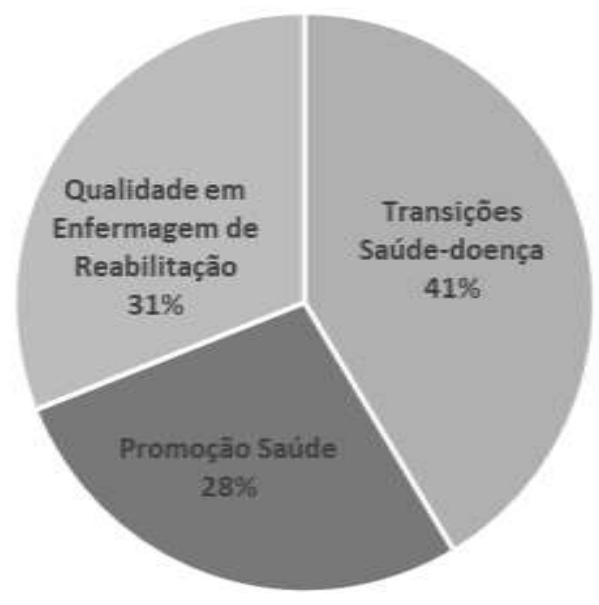

Figura 3 - Foco das temáticas dos estudos em análise.

Quadro 1 - Sumário dos estudos.

\begin{tabular}{|c|c|c|c|c|}
\hline Cód. & Referência & Objetivo do estudo & $\begin{array}{l}\text { Tipo de } \\
\text { estudo }\end{array}$ & Amostra \\
\hline E1 & Amaral ${ }^{15}$ & $\begin{array}{l}\text { Descrever o fenómeno de } \\
\text { adaptação após lesão } \\
\text { medular, enfatizando a } \\
\text { explicação das estratégias de } \\
\text { adaptação adotadas, e } \\
\text { procurando identificar as } \\
\text { implicações que têm nos } \\
\text { cuidados. }\end{array}$ & $\begin{array}{l}\text { Grounded } \\
\text { Theory }\end{array}$ & $\begin{array}{c}9 \\
\text { Indivíduos que } \\
\text { sofreram } \\
\text { lesão medular }\end{array}$ \\
\hline E2 & Azevedo, Gomes ${ }^{16}$ & $\begin{array}{lcc}\text { Determinar os efeitos } & \text { da } \\
\text { mobilização precoce } & \text { na } \\
\text { reabilitação funcional } & \text { de } \\
\text { doentes internados } & \text { em } \\
\text { cuidados intensivos. } & \end{array}$ & $\begin{array}{c}\text { Revisão } \\
\text { integrativa }\end{array}$ & $\begin{array}{l}6 \text { estudos com } \\
\text { doentes } \\
\text { internados em } \\
\text { cuidados } \\
\text { intensivos }\end{array}$ \\
\hline E3 & Cardoso et $\mathrm{al}^{17}$ & $\begin{array}{l}\text { Descrever os dados } \\
\text { relevantes do perfil dos } \\
\text { idosos de uma Unidade de } \\
\text { Cuidados na Comunidade } \\
\text { (UCC). }\end{array}$ & $\begin{array}{l}\text { Estudo } \\
\text { quantitativo, } \\
\text { descritivo e } \\
\text { exploratório }\end{array}$ & $\begin{array}{l}1.522 \text { idosos } \\
\text { vivendo no } \\
\text { seu domicílio }\end{array}$ \\
\hline E4 & Castro et al ${ }^{18}$ & $\begin{array}{l}\text { Avaliar o contributo da } \\
\text { terapia por caixa de espelho } \\
\text { para a autonomia no } \\
\text { autocuidado nos doentes com } \\
\text { hemiplegia/hemiparesia, por } \\
\text { AVC. }\end{array}$ & $\begin{array}{l}\text { Estudo quase- } \\
\text { experimental } \\
\text { com grupo } \\
\text { controlo não } \\
\text { equivalente }\end{array}$ & $\begin{array}{l}30 \text { doentes } \\
\text { vítimas de } \\
\text { AVC }\end{array}$ \\
\hline E5 & Cruz et al ${ }^{19}$ & $\begin{array}{l}\text { Traduzir e adaptar o } \\
\text { Hospitalized Elderly Needs } \\
\text { Awareness Scale } \text { (Henas) } \\
\text { para o português do Brasil e } \\
\text { o de Portugal. }\end{array}$ & $\begin{array}{l}\text { Estudo } \\
\text { quantitativo, } \\
\text { descritivo } \\
\text { correlacional }\end{array}$ & $\begin{array}{l}30 \text { pessoas } \\
\text { com lesão } \\
\text { medular } \\
\text { Traumática }\end{array}$ \\
\hline
\end{tabular}




\begin{tabular}{|c|c|c|c|c|}
\hline E6 & Faria et $\mathrm{a}^{20}$ & $\begin{array}{l}\text { Descrever o percurso da } \\
\text { pessoa com Acidente } \\
\text { Vascular Encefálico e } \\
\text { identificar os acontecimentos } \\
\text { significativos neste percurso. }\end{array}$ & $\begin{array}{l}\text { Estudo } \\
\text { Qualitativo } \\
\text { Exploratório- } \\
\text { descritivo }\end{array}$ & $\begin{array}{l}13 \text { pessoas } \\
\text { que se } \\
\text { tornaram } \\
\text { dependentes } \\
\text { devido à } \\
\text { Acidente } \\
\text { Vascular } \\
\text { Encefálico }\end{array}$ \\
\hline E7 & Ferreira et $\mathrm{al}^{21}$ & $\begin{array}{l}\text { Traduzir e adaptar para o } \\
\text { contexto português a escala } \\
\text { GUSS em doentes agudos e } \\
\text { avaliar as suas propriedades } \\
\text { psicométricas. }\end{array}$ & $\begin{array}{l}\text { Estudo } \\
\text { quantitativo, } \\
\text { descritivo } \\
\text { correlacional }\end{array}$ & $\begin{array}{l}174 \text { doentes } \\
\text { agudos }\end{array}$ \\
\hline E8 & Pereira et $\mathrm{al}^{22}$ & $\begin{array}{l}\text { Compreender as fragilidades } \\
\text { e/ou potencialidades das } \\
\text { respostas das autarquias } \\
\text { locais para abolir barreiras } \\
\text { arquitetónicas para pessoas } \\
\text { com mobilidade } \\
\text { condicionada. }\end{array}$ & $\begin{array}{c}\text { Estudo } \\
\text { descritivo, } \\
\text { exploratório }\end{array}$ & $\begin{array}{l}21 \\
\text { presidentes } \\
\text { de junta }\end{array}$ \\
\hline E9 & Fernandes et $\mathrm{al}^{23}$ & $\begin{array}{l}\text { Avaliar o impacto de um jogo } \\
\text { para promover a educação } \\
\text { postural das crianças. }\end{array}$ & $\begin{array}{l}\text { Estudo } \\
\text { quantitativo, } \\
\text { descritivo } \\
\text { correlacional }\end{array}$ & $\begin{array}{l}215 \text { crianças } \\
\text { do ensino pré- } \\
\text { escolar e do } \\
1^{\circ} \text { ciclo do } \\
\text { ensino básico }\end{array}$ \\
\hline E10 & Fonseca et $\mathrm{al}^{24}$ & $\begin{array}{l}\text { Identificar indicadores } \\
\text { sensíveis aos cuidados de } \\
\text { enfermagem de reabilitação, } \\
\text { ao nível do autocuidado, em } \\
\text { relação às pessoas com } \\
\text { patologia respiratória. }\end{array}$ & $\begin{array}{l}\text { Revisão } \\
\text { sistemática da } \\
\text { literatura }\end{array}$ & $\begin{array}{l}6 \text { estudos com } \\
\text { pessoas com } \\
\text { patologia } \\
\text { respiratória }\end{array}$ \\
\hline E11 & Fonseca et $\mathrm{al}^{25}$ & $\begin{array}{l}\text { Identificar indicadores } \\
\text { sensíveis aos cuidados de } \\
\text { enfermagem de reabilitação, } \\
\text { ao nível do autocuidado, em } \\
\text { relação às pessoas em } \\
\text { processo cirúrgico. }\end{array}$ & $\begin{array}{l}\text { Revisão } \\
\text { sistemática da } \\
\text { literatura }\end{array}$ & $\begin{array}{l}11 \text { estudos } \\
\text { com pessoas } \\
\text { adultas em } \\
\text { processo } \\
\text { cirúrgico }\end{array}$ \\
\hline E12 & Fonseca et $\mathrm{al}^{26}$ & $\begin{array}{l}\text { Determinar os indicadores a } \\
\text { incluir na ontologia do } \\
\text { envelhecimento, em relação } \\
\text { aos cuidados de enfermagem } \\
\text { de reabilitação em relação às } \\
\text { pessoas idosas dependentes } \\
\text { no autocuidado com várias } \\
\text { comorbilidades. }\end{array}$ & $\begin{array}{l}\text { Revisão } \\
\text { sistemática da } \\
\text { literatura }\end{array}$ & $\begin{array}{c}11 \text { estudos } \\
\text { com pessoas } \\
\text { dependentes } \\
\text { no } \\
\text { autocuidado }\end{array}$ \\
\hline E13 & Fumincelli et $\mathrm{al}^{27}$ & $\begin{array}{l}\text { Medir e comparar a } \\
\text { qualidade de vida da pessoa } \\
\text { com bexiga neurogénica e } \\
\text { utilização do cateterismo } \\
\text { urinário intermitente em } \\
\text { processo de reabilitação, no } \\
\text { Brasil e em Portugal. }\end{array}$ & \begin{tabular}{|} 
Estudo \\
multicêntrico, \\
quantitativo, \\
descritivo e \\
correlacional.
\end{tabular} & $\begin{array}{c}170 \text { doentes } \\
\text { Brasileiros e } \\
52 \\
\text { Portugueses }\end{array}$ \\
\hline E14 & Gomes B ${ }^{28}$ & $\begin{array}{l}\text { Identificar os contributos da } \\
\text { formação dos profissionais de } \\
\text { enfermagem na área da }\end{array}$ & $\begin{array}{l}\text { Estudo } \\
\text { quantitativo, } \\
\text { descritivo }\end{array}$ & $\begin{array}{c}39 \\
\text { enfermeiros } \\
\text { de unidades }\end{array}$ \\
\hline
\end{tabular}




\begin{tabular}{|c|c|c|c|c|}
\hline & & $\begin{array}{l}\text { Licenciatura e Especialização } \\
\text { em } \quad \text { Enfermagem r de } \\
\text { Reabilitação para ra } \\
\text { desenvolvimento das práticas } \\
\text { de cuidados. }\end{array}$ & & de saúde \\
\hline E15 & Gomes et $\mathrm{al}^{29}$ & $\begin{array}{l}\text { Identificar fatores que } \\
\text { contribuam para a qualidade } \\
\text { dos cuidados de enfermagem } \\
\text { de reabilitação em unidades } \\
\text { de internamento. }\end{array}$ & $\begin{array}{l}\text { Estudo } \\
\text { Qualitativo } \\
\text { Exploratório- } \\
\text { descritivo }\end{array}$ & $\begin{array}{c}12 \\
\text { enfermeiros } \\
\text { especialistas } \\
\text { em } \\
\text { Enfermagem } \\
\text { de } \\
\text { Reabilitação }\end{array}$ \\
\hline E16 & Mendes et $\mathrm{al}^{30}$ & $\begin{array}{l}\text { Descrever os diferentes } \\
\text { modelos de prestação de } \\
\text { cuidados de reabilitação em } \\
\text { prática nas unidades de } \\
\text { cuidados intensivos. }\end{array}$ & $\begin{array}{c}\text { Estudo } \\
\text { descritivo, } \\
\text { exploratório }\end{array}$ & $\begin{array}{l}\text { Enfermeiros- } \\
\text { chefes ou } \\
\text { responsáveis } \\
\text { das } 58 \\
\text { unidades de } \\
\text { cuidados } \\
\text { intensivos }\end{array}$ \\
\hline E17 & Gouveia et $\mathrm{al}^{31}$ & $\begin{array}{l}\text { Avaliar a eficácia do } \\
\text { programa de reabilitação do } \\
\text { ProBalance em HRQL de } \\
\text { idosos residentes r na } \\
\text { comunidade com deficiências } \\
\text { no equilíbrio. }\end{array}$ & $\begin{array}{l}\text { Estudo } \\
\text { randomizado } \\
\text { com grupo de } \\
\text { controle }\end{array}$ & 52 idosos \\
\hline E18 & Gouveia et $\mathrm{al}^{32}$ & $\begin{array}{l}\text { Avaliar o efeito de um } \\
\text { programa de reabilitação } \\
\text { liderado por enfermeiros } \\
\text { ProBalance) sobre o } \\
\text { equilíbrio eo risco de queda } \\
\text { dos idosos da comunidade. }\end{array}$ & $\begin{array}{l}\text { Estudo } \\
\text { randomizado } \\
\text { com grupo } \\
\text { de controlo }\end{array}$ & 52 idosos \\
\hline E19 & $\begin{array}{l}\text { Gouveia, Jardim, } \\
\text { Martins }^{33}\end{array}$ & $\begin{array}{l}\text { (1) Descrever as relações } \\
\text { entre a postura, a marcha e } \\
\text { a capacidade funcional dos } \\
\text { idosos e a cinesioterapia. } \\
\text { (2) Identificar referências à } \\
\text { intervenção do enfermeiro } \\
\text { especialista em reabilitação } \\
\text { neste domínio. }\end{array}$ & $\begin{array}{l}\text { Revisão } \\
\text { sistemática } \\
\text { da literatura }\end{array}$ & 11 estudos \\
\hline E20 & Luís ${ }^{34}$ & $\begin{array}{l}\text { Enunciar os problemas que } \\
\text { acompanham a hemiplegia, } \\
\text { salientar alguns aspetos de } \\
\text { atuação do enfermeiro de } \\
\text { reabilitação. }\end{array}$ & Não aplicável & $\begin{array}{l}\text { Doente } \\
\text { hemiplégico }\end{array}$ \\
\hline E21 & $\begin{array}{l}\text { Malheiro, Gaspar, } \\
\text { Barros }^{35}\end{array}$ & $\begin{array}{l}\text { Desenvolver e avaliar um } \\
\text { programa educacional que } \\
\text { promova a independência } \\
\text { funcional e competências de } \\
\text { autogestão em pessoas com } \\
\text { espinha bífida. }\end{array}$ & $\begin{array}{l}\text { Estudo } \\
\text { quase- } \\
\text { experimental }\end{array}$ & $\begin{array}{l}56 \\
\text { adolescentes } \\
\text { com espinha } \\
\text { bífida, com } \\
\text { idades entre } \\
10 \text { e } 18 \text { anos }\end{array}$ \\
\hline E22 & $\begin{array}{l}\text { Nunes, Queirós, } \\
\text { Paulo }^{36}\end{array}$ & $\begin{array}{l}\text { Analisar as estratégias } \\
\text { adotadas no planeamento } \\
\text { da alta do doente com } \\
\text { acidente vascular cerebral. }\end{array}$ & $\begin{array}{c}\text { Revisão } \\
\text { sistemática } \\
\text { da literatura }\end{array}$ & 19 estudos \\
\hline
\end{tabular}




\begin{tabular}{|c|c|c|c|c|}
\hline E23 & $\begin{array}{l}\text { Rodrigues, } \\
\text { Henriques }^{37}\end{array}$ & 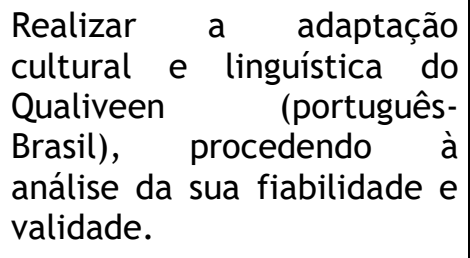 & $\begin{array}{l}\text { Estudo } \\
\text { quantitativo, } \\
\text { descritivo } \\
\text { correlacional }\end{array}$ & 72 indivíduos \\
\hline E24 & Rosa, Martins ${ }^{38}$ & $\begin{array}{l}\text { Identificar os elementos a } \\
\text { integrar num Programa de } \\
\text { Enfermagem de Reabilitação } \\
\text { Sensório Motora (PERSM) } \\
\text { destinado a Recém-Nascidos } \\
\text { Pré-Termo (RNPT). }\end{array}$ & $\begin{array}{l}\text { Revisão } \\
\text { sistemática } \\
\text { da literatura }\end{array}$ & 7 estudos \\
\hline E25 & Prado et $\mathrm{al}^{39}$ & $\begin{array}{l}\text { Agregar o que foi produzido } \\
\text { em relação ao banho como } \\
\text { procedimento relacionado } \\
\text { ao cuidado de enfermagem } \\
\text { e ao diagnóstico de doentes } \\
\text { dependentes submetidos a } \\
\text { processos de reabilitação } \\
\text { após um evento traumático. }\end{array}$ & $\begin{array}{l}\text { Estudo de } \\
\text { revisão }\end{array}$ & 10 estudos \\
\hline E26 & Preto et a ${ }^{40}$ & $\begin{array}{l}\text { Avaliar mudanças na aptidão } \\
\text { funcional de idosos } \\
\text { institucionalizados através } \\
\text { de um programa de } \\
\text { enfermagem de reabilitação } \\
\text { baseado em exercício físico } \\
\text { de moderada intensidade. }\end{array}$ & $\begin{array}{l}\text { Pré- } \\
\text { teste/pós- } \\
\text { teste com } \\
\text { grupo de } \\
\text { controlo. }\end{array}$ & 178 idosos \\
\hline E27 & Garcia, Araújo ${ }^{41}$ & $\begin{array}{l}\text { Avaliar o impacto de um } \\
\text { instrumento vídeo, em } \\
\text { português, sobre técnicas } \\
\text { de autocuidado e } \\
\text { reabilitação motora em } \\
\text { pessoas com Lesão Medular } \\
\text { (LM). }\end{array}$ & $\begin{array}{l}\text { Estudo quasi- } \\
\text { experimental }\end{array}$ & $\begin{array}{l}60 \\
\text { participantes }\end{array}$ \\
\hline E28 & Preto et $\mathrm{al}^{42}$ & $\begin{array}{l}\text { Avaliar, no pré-operatório e } \\
\text { aos dois meses pós-cirurgia, } \\
\text { a gravidade dos sintomas e } \\
\text { o estado funcional, através } \\
\text { do Questionário de Boston } \\
\text { (QB) e a qualidade de vida } \\
\text { através do SF-36 v2. }\end{array}$ & $\begin{array}{l}\text { Estudo } \\
\text { prospetivo }\end{array}$ & $\begin{array}{l}50 \text { doentes } \\
\text { submetidos a } \\
\text { cirurgia }\end{array}$ \\
\hline E29 & Fernandes et $\mathrm{al}^{43}$ & $\begin{array}{l}\text { Mapear a produção } \\
\text { científica sobre a utilização } \\
\text { da caminhada enquanto } \\
\text { intervenção nos utentes } \\
\text { sujeitos a quimioterapia. }\end{array}$ & $\begin{array}{l}\text { Estudo } \\
\text { revisão }\end{array}$ & 7 estudos \\
\hline
\end{tabular}

